\title{
Muscle Fatigue Affects Mental Simulation of Action
}

\author{
Laurent Demougeot, ${ }^{1,2}$ and Charalambos Papaxanthis ${ }^{1,2}$ \\ ${ }^{1}$ Université de Bourgogne, Unité de Formation et de Recherche en Sciences et Techniques des Activité Physiques et Sportives, F-21078 Dijon, France, and \\ 2Institut National de la Santé et de la Recherche Médicale (INSERM), Unité 887, Motricité et Plasticité, F-21078 Dijon, France
}

Several studies suggest that when subjects mentally rehearse or execute a familiar action, they engage similar neural and cognitive operations. Here, we examined whether muscle fatigue could influence mental movements. Participants mentally and actually performed a sequence of vertical arm movements (rotation around the shoulder joint) before and after a fatiguing exercise involving the right arm. We found similar durations for actual and mental movements before fatigue, but significant temporal discrepancies after fatigue. Specifically, mental simulation was accelerated immediately after fatigue, while the opposite was observed for actual execution. Furthermore, actual movements showed faster adaptation (i.e., return to prefatigue values) than mental movements. The EMG analysis showed that postfatigue participants programmed larger, compared to prefatigue, neural drives. Therefore, immediately after fatigue, the forward model received dramatically greater efferent copies and predicted faster, compared to prefatigue, arm movements. During actual movements, the discrepancy between estimated (forward model output) and actual state (sensory feedback) of the arm guided motor adaptation; i.e., durations returned rapidly to prefatigue values. Since during mental movements there is no sensory information and state estimation derives from the forward model alone, mental durations remained faster after fatigue and their adaptation was longer than those of actual movements. This effect was specific to the fatigued arm because actual and mental movements of the left nonfatigued arm were unaffected. The current results underline the interdependence of motor and cognitive states and suggest that mental actions integrate the current state of the motor system.

\section{Introduction}

Humans are able to predict their interaction with the external environment by mentally simulating their actions. Neuroimaging and behavioral studies suggest that when subjects mentally rehearse or execute a familiar action, they engage similar neural and cognitive operations. For instance, mental and actual movements share overlapping neural substrates (Jeannerod, 2001; Fadiga and Craighero, 2004; Munzert et al., 2009) and preserve the same spatiotemporal characteristics (Courtine et al., 2004; Bakker et al., 2007; Gueugneau et al., 2009). Furthermore, appropriate mental training can enhance motor performance (Ranganathan et al., 2004; Gentili et al., 2006, 2010). Forward internal models are in the core of actual movements and provide an interesting theoretical basis for interpreting their behavioral similarities with mental movements (Wolpert and Flanagan, 2001). Forward models mimic the causal flow of the physical process by predicting the consequences (e.g., position, velocity) of a motor command. During mental actions, the CNS prepares, but retains, motor commands before they reach the neuromuscular level. Consequently, although no overt movement occurs, a corollary discharge (i.e., efference copy) and the current state are both

Received Nov. 18, 2010; revised May 27, 2011; accepted June 1, 2011.

Author contributions: C.P. designed research; L.D. performed research; L.D. and C.P. analyzed data; L.D. and C.P. wrote the paper.

We thank Boris Matkowski for his advice about muscle fatigue, as well as Nicolas Schweighofer and Lina Papamichail for their valuable comments on the manuscript.

Correspondence should be addressed to Charalambos Papaxanthis at the above address. E-mail: charalambos.papaxanthis@u-bourgogne.fr.

DOI:10.1523/JNEUROSCI.6032-10.2011

Copyright $\odot 2011$ the authors $\quad 0270-6474 / 11 / 3110712-09 \$ 15.00 / 0$ available to the forward model, which provides future state estimations. State estimation during mental training generates an internal training signal (Wolpert et al., 1995; Kawato, 1999) that enhances the performance of upcoming movements (Gentili et al., 2010).

A central question is to what extent modifications at the sensorimotor state could influence the content of mental actions. For instance, we have all experienced muscle fatigue after a demanding day, and athletes are regularly facing the challenge of muscle fatigue during competitions. Muscle fatigue reduces maximal force (Gandevia, 2001), affects movement coordination (Schmid et al., 2006), and alters movement or effort senses (Walsh et al., 2010). While abundant data exist for actual movements, the effects of muscle fatigue on mental movements have not yet been explored. Does muscle fatigue alter mental movement simulation? Is that effect general, or is it restricted to mental movements that engage the fatigued limbs only? In this study, we measured the temporal features of actual and mental movements before and after muscle fatigue. The analysis of the temporal aspects of mental actions becomes a potential tool to investigate action representation (Munzert et al., 2009; Gentili et al., 2010). A strong prediction is that duration of mental actions should be sensitive to muscle fatigue, because the forward model receives as input the efferent copy of inappropriate motor commands and the perturbed state of the arm, both caused by muscle fatigue. Because mental actions are effector specific (Ehrsson et al., 2003), we expected that this effect would be restricted to the fatigued arm without being transferred to the nonfatigued arm. We also assumed that recovering from fatigue should be longer for mental than actual movements. In actual movements, the sensory inflow 
A

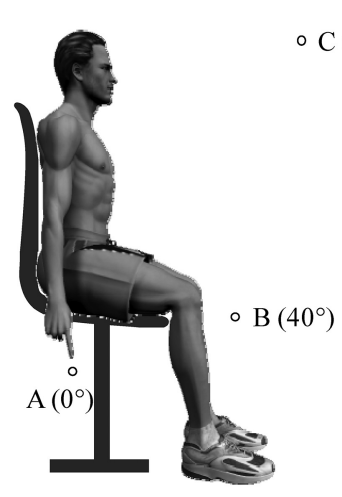

B

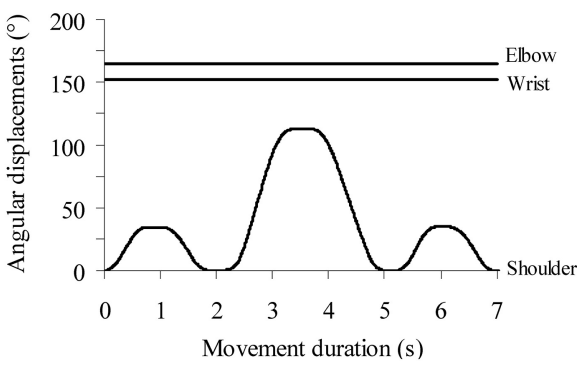

E

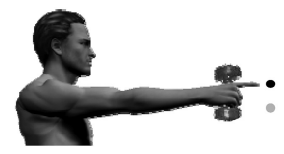

C

\begin{tabular}{|l||l|}
\hline \multicolumn{1}{|c||}{ Session Ia } & \multicolumn{1}{|c|}{ Session IIa } \\
\hline Pre-Fatigue (mental right arm) & Pre-Fatigue (actual right arm) \\
Fatiguing exercise (right arm) & Fatiguing exercise (right arm) \\
Post-Fatigue (mental right arm) & Post-Fatigue (actual right arm) \\
\hline
\end{tabular}

D Left arm movements

\begin{tabular}{|l||c|}
\hline \multicolumn{1}{|c|}{ Session Ib } & \multicolumn{1}{|c|}{ Session IIb } \\
\hline $\begin{array}{l}\text { Pre-Fatigue (mental left arm) } \\
\text { Fatiguing exercise (right arm) } \\
\text { Post-Fatigue (mental left arm) }\end{array}$ & Pre-Fatigue (actual left arm) \\
\hline
\end{tabular}

Figure 1. A, Participants' initial position and spatial location of the targets during right arm movements (right-side view). Participants actually or mentally pointed between the three targets following a specific order (A-B-A-C-A-B-A). Actual and mental movements were performed with the right and the left arm. $\boldsymbol{B}$, Typical angular displacements of the shoulder, elbow, and wrist joints in the sagittal plane (elevation angles). $\boldsymbol{C}, \boldsymbol{D}$, Experimental procedures for the right and the left arm movements. Right $(\boldsymbol{C})$ and left (D) arm movements were counterbalanced between participants. Mental movements (session I) were always performed before actual movements (session II), because we wanted participants not to have explicit knowledge about the effects of muscle fatigue on motor performance. $E$, Fatiguing task. Participants had to maintain their right arm in the horizontal plane for as long as possible. Two targets in the sagittal plane indicated the initial (black) and the final-tolerated (gray) arm position.

is combined with the output of the forward model to provide accurate state estimations (Miall et al., 1993; Wolpert and Ghahramani, 2000), while in mental movements, the state estimation derives from the forward model alone.

\section{Materials and Methods Participants}

Seventeen adults (mean age $=28.1 \pm 2.5 \mathrm{SD}, 12$ males and 5 females) volunteered to participate in the experiments described in this study. They had no history of neurological disorders and had normal or corrected-to-normal vision. Their motor imagery ability was assessed by the French version of the Movement Imagery Questionnaire "MIQr" (Hall and Martin, 1997). All were good imagers, as they obtained scores $>40$ (maximum score 56). Hand preference was evaluated by the Edinburgh test (Oldfield, 1971), which showed that all the participants were strongly right handed (individual scores $>0.87$ ). The regional ethics committee of Burgundy (C.E.R.) approved the experimental protocol, which was performed in agreement with local requirements and international norms (Declaration of Helsinki, 1964).

\section{Main experiment \\ Motor tasks}

The experiments took place in a quiet room under good lighting conditions to ensure that participants were able to see their arm and the experimental device. They were comfortably sat on a chair with their trunk aligned in the vertical position being supported by the back of the chair. Three targets were centered on their right or left shoulder (parasagittal plane) in a polar frame of reference at a distance equal to the length of their fully extended arm (Fig. 1A). The elevation angle of the shoulder joint when the index fingertip pointed toward targets $\mathrm{A}, \mathrm{B}$, and $\mathrm{C}$ was $0^{\circ}, 40^{\circ}$, and $110^{\circ}$, respectively. Participants were requested to execute (actual) or to imagine (mental) arm pointing movements between the three targets. Actual and mental movements were performed with the right and the left arm before and after a fatiguing exercise. During both actual and mental trials, participants had their eyes open. An actual or a mental trial started with the arm aligned with the vertical axis (hand downward) and consisted of six subsequent arm movements between the targets (A-B-AC-A-B-A). To obtain valid and reliable measurements in motor imagery protocol, several movements are necessary because of the short duration of a single movement and the course resolution of mental movement time measurements (Sirigu et al., 1996; Skoura et al., 2008; Gentili et al., 2010). Actual arm movements mobilized only the shoulder joint (Fig. $1 B$ ). The shoulder angular displacement was $40^{\circ}$ between targets $\mathrm{A}$ and $\mathrm{B}$, and $110^{\circ}$ between targets A and C. During actual movements, the elbow joint was fully extended and the semipronated hand was aligned with the upper arm and the forearm. The participants were requested to perform actual and mental arm movements at a natural self-selected speed. We particularly instructed participants to mentally simulate arm movements in a first perspective (kinesthetic or motor imagery, as in Demougeot et al., 2009). Eye movements were tolerated during both mental and actual movements to facilitate the fixation of the targets during the motion of the arm (as in Gueugneau et al., 2008). During mental movements, the arm remained immobile and aligned with the vertical axis. After the achievement of the experimental protocol, none of the participants reported difficulties in internally simulating arm movements in a first perspective.

\section{Experimental protocol}

In the main experiment, eight participants (mean age $=28.9 \pm 2.5 \mathrm{SD}, 5$ males and 3 females) performed actual and mental movements with their right and left arm before and after a fatiguing exercise (see below). Right and left arm movements were counterbalanced between participants and were performed with a 2 week time interval. Within each block of right or left arm movements, the session of mental movements was always performed first, because we wanted participants not to have explicit knowledge about the effects of muscle fatigue on motor performance.

Right arm movements. Participants performed mental (session Ia) as well as actual (session IIa) vertical right arm movements before and after a fatiguing task involving the right arm (Fig. 1C). They accomplished the mental arm movements first, and after a week, they accomplished the actual arm movements. Session Ia consisted of a prefatigue test (12 mental right arm movements), a fatiguing exercise involving the right arm, and a postfatigue test (20 mental right arm movements). Session IIa consisted of a prefatigue test (12 actual right arm movements), a fatiguing exercise involving the right arm, and a postfatigue test ( 20 actual right arm movements).

Left arm movements. Participants performed mental (session Ib) as well as actual (session IIb) left arm movements before and after a fatiguing task involving the right arm (Fig. $1 D$ ). They accomplished the mental arm movements first, and after a week, they accomplished the actual arm movements. The aim of this condition was to investigate whether fatigue of the right arm has specific (i.e., right arm fatigue influences the mental and actual performance of right arm movements) or general (i.e., right arm fatigue influences the mental and actual performance of left arm movements) effects. The session Ib consisted of a prefatigue test (12 mental left arm movements), a fatiguing exercise involving the right arm, and a postfatigue test (20 mental left arm movements). The session IIb consisted of a prefatigue test (12 actual left arm movements), a fatiguing exercise involving the right arm, and a postfatigue test ( 20 actual left arm movements). 
For the four sessions, the postfatigue trials started immediately after the fatiguing exercise. The intertrial time interval in the prefatigue and the postfatigue tests was $\sim 2 \mathrm{~s}$. Before the prefatigue test, participants were familiarized with the motor task by realizing three actual and three mental movements with their right and left arm.

Fatiguing exercise. Participants had to maintain their fully extended right arm in the horizontal plane (isometric contraction) for as long as possible while holding a mass in the palm of their hand (Fig. 1E). They were orally encouraged by the experimenters to pursue the task until exhaustion. The task stopped when the participants' fatigued right arm reached a limit position, which corresponded to a downward shoulder angular displacement of $5^{\circ}$ (Fig. $1 E$ ). The adding mass was adapted to the participants' right shoulder force level. More specifically, the adding mass that would provide a shoulder gravitational torque (measured with the arm at the horizontal position) equal to $50 \%$ of the maximum torque exerted by the flexor muscles of the right shoulder joint was calculated. The maximum torque generated by the flexor muscles of the right shoulder joint (the arm was placed at the horizontal position) was evaluated by means of Biodex isokinetic dynamometer. Participants performed three maximal voluntary isometric contractions (MVCs). Each MVC lasted 5-6 s, and a resting time of 2 min was taken between each trial to avoid any effect of muscle fatigue on the measurements.

EMG data acquisition. EMG signals were recorded by means of two silver-chloride surface electrodes of $10 \mathrm{~mm}$ diameter placed on the belly muscle (with the skin previously shaved and cleaned) with an interelectrode distance (center to center) of $2 \mathrm{~cm}$. The EMG activity was recorded from the following muscles of the participants' right and left arms: deltoid anterior (DA), deltoid posterior (DP), biceps brachii (BB), triceps brachii medial head (TBM), and triceps brachii long head (TBL). The reference electrode was placed on the inferior side of the left or right wrist. EMG signals were acquired with a sampling frequency of $2 \mathrm{kHz}$ and processed with a multi channel analog-digital converter (Biopac Systems). EMG signals were filtered with a bandwidth frequency ranging from $20 \mathrm{~Hz}$ to $400 \mathrm{~Hz}$, and amplified with a gain of 1000 .

Kinematic data acquisition. Right and left actual arm movements were recorded by using 6 TV cameras of an optoelectronic system of motion analysis (SMART, BTS). Kinematic signals were acquired with a sampling frequency of $120 \mathrm{~Hz}$. Four reflective markers were placed on the shoulder (acromion), elbow (lateral epicondyle), wrist (styloid process of the wrist), and the nail of the index fingertip. Kinematic parameters (movement duration and peak velocity) in three dimensions $(x, y$, and $z$ ) were low-pass filtered using a digital fifth-order Butterworth filter at a cutoff frequency of $10 \mathrm{~Hz}$. Finger movement onset was defined as the moment at which linear tangential velocity of the index fingertip exceeded $5 \%$ of its peak and the end of movement as the point at which the same velocity dropped below the $5 \%$ threshold.

Quantification of muscle fatigue. To evaluate the level of muscle fatigue during the fatiguing exercise, we computed every $10 \mathrm{~ms}$ the root mean square (RMS) and the median frequency (MF) of the EMG signals during two time periods: the first $10 \mathrm{~s}$ and the last $10 \mathrm{~s}$ of the fatiguing task.

RMS was calculated using the following formula:

$$
\mathrm{RMS}=\sqrt{\frac{1}{\mathrm{MD}} \int_{0}^{\mathrm{MD}}(\mathrm{EMG})^{2} d t}
$$

where MD is the movement duration.

RMS and MF parameters are both sensible to the development of muscular fatigue. Previous studies have shown that RMS values increased and MF values decreased during a fatiguing exercise (Komi and Tesch, 1979; Jurell, 1998). We expected similar results between the first $10 \mathrm{~s}$ and the last $10 \mathrm{~s}$ of our fatiguing task. We verified that RMS and MF values showed normal distribution (Shapiro-Wilk tests). Consequently, we compared for each muscle and each parameter (i.e., RMS and MF) the first $10 \mathrm{~s}$ with the last $10 \mathrm{~s}$ of the fatiguing task by performing two-tailed paired $t$ test (level of significance $p<0.05)$.
In a preliminary study, which involved five male adults (mean age $=$ $25.7 \pm 3.1 \mathrm{SD}$ ) who did not participate in the experiments, we measured the maximum force immediately after the fatiguing task. We found that our fatiguing protocol induced a significant decrease of maximum muscle force (on average $=-20.5 \pm 3.5 \% ; t=7.93, \mathrm{df}=4, p=0.004$ ). In this preliminary study, we observed that measuring maximum force after the fatiguing exercise delayed the beginning of actual and mental postfatigue trials and reduced participants' concentration. Therefore, to preserve the plan of our protocol (i.e., the timing between the prefatigue trials, the fatigue exercise, and the postfatigue trials), and the quality of mental movement simulation, we decided not to measure the participants' maximum force after the fatiguing task in the main experimental protocol, and to quantify muscle fatigue by analyzing the EMG pattern (see above).

EMG analysis during mental arm movements. We examined whether muscle activation was present during mental arm movements. Before mental sessions Ia and Ib, we determined the baseline level of the EMG signal (no voluntary muscle activation) by recording the EMG activity of the totally relaxed arm muscles (five trials of $7 \mathrm{~s}$ ). We calculated the average RMS value during the rest phase and compared it (trial-by-trial comparison) with the RMS values of each trial in prefatigue and postfatigue tests. As RMS values showed normal distribution (Shapiro-Wilk tests), we performed two-tailed paired $t$ test comparisons (level of significance $p<0.05$ ).

EMG analysis during actual movements. EMG signals during the prefatigue and the postfatigue actual trials were cut off $100 \mathrm{~ms}$ before movement onset and $100 \mathrm{~ms}$ after movement offset. Typical biphasic patterns of agonist and antagonist muscles were seen in all muscles. We calculated the following parameters on the EMG patterns: the duration of agonist and antagonist bursts, the time to peak agonist and antagonist bursts, the time of initiation of antagonist burst, and the RMS of the agonist bursts. Onset and offset of muscle activities was defined by a $5 \%$ threshold of their peak values. RMS was calculated every $10 \mathrm{~ms}$.

Recording and statistical analysis of the duration of actual and mental trials. Movement duration is the only variable that can be measured during mental movements and compared with actual movements. Durations of actual and mental arm movements were recorded by means of an electronic stopwatch (temporal resolution $1 \mathrm{~ms}$ ). The experimenter started the stopwatch at the moment of the vocal signal "go" (onset of the mental or actual arm movement) and stopped it when the participants indicated orally the offset of the actual or the mental arm movement (i.e., vocal signal "stop"). To test the reliability of measuring movement duration with a stopwatch, we compared, trial by trial, the actual movement durations recorded by the stopwatch with the actual movement durations recorded by the optoelectronic system. This comparison was performed for both the right and the left arm movements. We did not find significant differences, neither for the prefatigue (12 trials) nor for the postfatigue (20 trials) tests (one-way ANOVA; $p>0.05$ ).

For the comparison of actual and mental trials, we used the durations recorded by the stopwatch. These durations showed normal distribution (Shapiro-Wilk tests) in both the prefatigue and postfatigue tests. We performed a repeated-measures ANOVA with two factors: movement type (actual, mental) and trials (12 prefatigue and 20 postfatigue). Statistical analysis was performed for the right and the left arm separately. Post hoc comparisons were performed by means of Newman-Keuls tests. For all the statistical analysis, the level of significance was fixed at $p<0.05$.

Postfatigue adaptation. For both actual and mental trials, we calculated the duration of postfatigue adaptation, i.e., the time needed to return to prefatigue values after the end of the fatiguing exercise. This duration was calculated from the end of the fatiguing exercise to the end of the last postfatigue trial (actual or mental) that was significantly different from the prefatigue average value. Durations showed normal distribution (Shapiro-Wilk tests) and differences between mental and actual trials were assessed by means of two-tailed paired $t$ test (level of significance $p<0.05)$.

\section{Control experiments}

In the first control experiment, we examined whether postfatigue adaptation of mental arm movements (i.e., the time needed to return to prefatigue values after the fatiguing exercise) evolved in parallel with a 


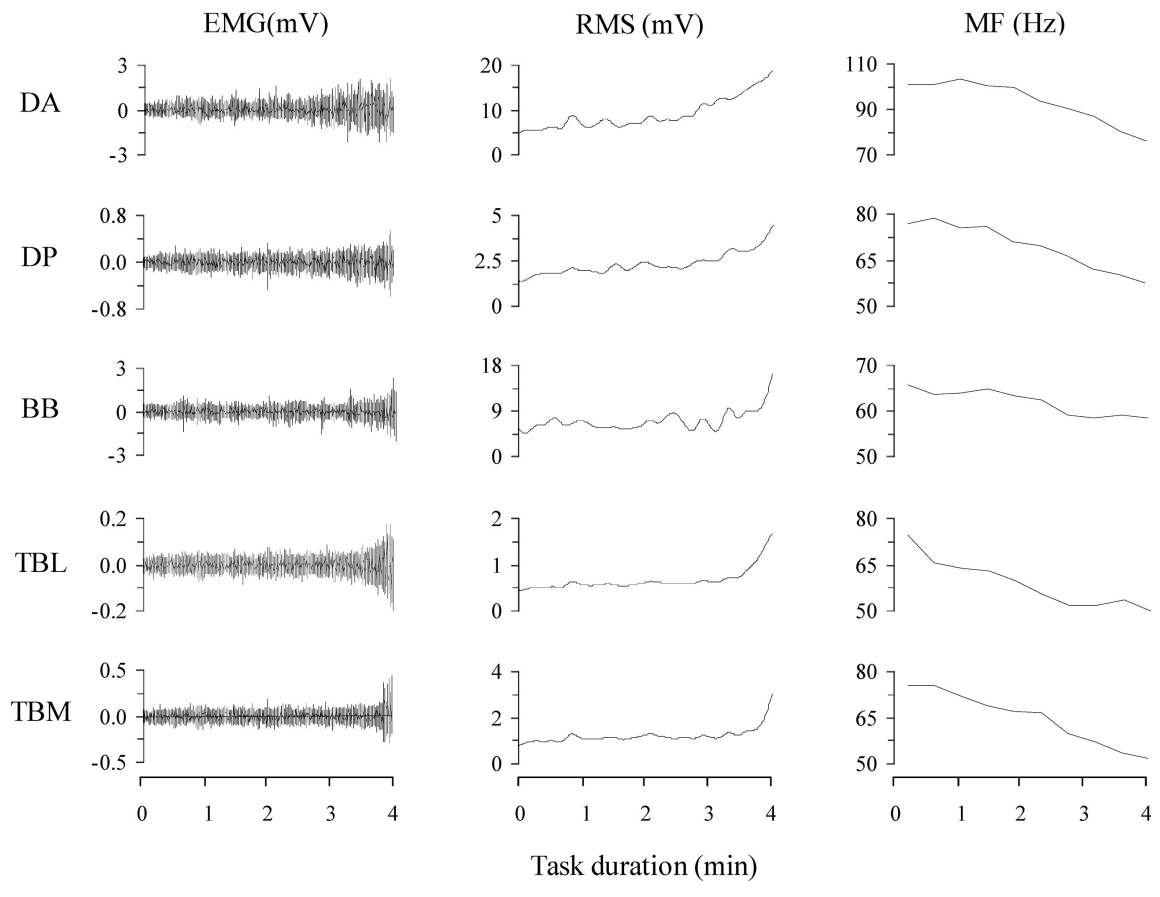

Figure 2. The fist column illustrates typical EMG patterns from one participant during the fatiguing task. The second and the third columns show, respectively, the RMS and the MF of the EMG signals.

natural recovering from fatigue. The same subjects participated in this experiment, which comprised three sessions separated by a period of 1 week. Each session consisted of a prefatigue test, a fatiguing exercise, and a postfatigue test. The three sessions differed by the time of administration of the postfatigue test after the fatiguing exercise. In the prefatigue test, participants maintained their right arm in the horizontal position for $5 \mathrm{~s}$ (five repetitions). In the fatiguing exercise, the participants' right arm was fatigued by the same procedures described in the Materials and Methods section of the main experiment. The postfatigue test was identical to prefatigue test, with the difference that it was realized at three different time intervals after the end of the fatiguing exercise: short time interval (session I), medium time interval (session II), and long time interval (session III). During these intervals, the participants relaxed their right arm to recover from fatigue. These three time intervals were calculated for each participant individually and corresponded to the time interval between the end of the fatiguing exercise and the end of each of the first three postfatigue mental trials (see Figs. $1 D, 3 A$ ). The rest period was on average $10.2 \mathrm{~s}$ for the short time interval, $18.5 \mathrm{~s}$ for the medium time interval, and $27.3 \mathrm{~s}$ for the long time interval. We recorded and analyzed the EMG activity from the arm muscles as described in the Materials and Methods section of the main experiment. We compared, by means of $t$ tests, the RMS and MF values between the prefatigue and the three postfatigue tests (short, medium, and long time intervals) to examine the time course of natural recovering from fatigue.

In the second control experiment, we verified that postfatigue adaptation was qualitatively similar between actual and mental trials. In the main experiment, we examined postfatigue adaptation by considering the duration of the whole trial (mental or actual). An intriguing question, however, is related to the adaptation of each of the six arm movements that composed each trial. However, while a movement-by-movement analysis is plausible for actual trials, it is not for mental trials (during motor imagery no movement occurs). To indirectly verify that postfatigue adaptation was qualitatively similar between actual and mental trials, we asked five new participants (mean age $=27.6 \pm 2 \mathrm{SD}, 3$ males and 2 females) to carry out the following protocol, which included two sessions. The session I consisted of a prefatigue test (12 actual trials with the right arm), a fatiguing exercise (similar to the main experiment), and a postfatigue test ( 4 mental and one actual trial). The session II consisted of a prefatigue test (12 actual trials with the right arm), a fatiguing exer- cise (similar to the main experiment), and a postfatigue test ( 5 actual trials). The two sessions were performed with a $2 \mathrm{~d}$ time interval. We recorded arm kinematics and EMG activity from arm muscles as described in the Materials and Methods section. We compared between them the first prefatigue, the first postfatigue, and the fifth postfatigue trials. Due to the small number of participants $(n=5)$, statistical differences were accessed by means of nonparametric Wilcoxon tests.

In the third control experiment, we wanted to rule out the possibility that temporal differences between prefatigue and postfatigue actual or mental trials, observed in the main experiment, were due to variations in arm postures. Indeed, the arm was motionless in the horizontal axis during fatigue, motionless in the vertical axis during mental trials, while it was displaced during actual trials. Eight participants (mean age $=28 \pm 2.4 \mathrm{SD}, 6$ males and 2 females, four of them took place in the first experiment) volunteered to carry out the sessions Ia and $\mathrm{Ib}$ of the main experiment. The unique variation between the control and the main experiment was the replacement of the fatiguing task by a passive task, in which the right arm was passively maintained at horizontal level. The session Ia consisted of a pretest (12 mental right arm movements), a passive posture involving the right arm (arm fully extended and supported by a device at the horizontal plane), and a posttest (20 mental right arm movements). The session Ib consisted of a pretest test (12 actual right arm movements), a passive posture involving the right arm (arm fully extended and supported by a device at the horizontal plane), and a posttest test (20 actual right arm movements). Each participant rested in the passive position for a duration that was equivalent to the average duration of the fatiguing task in the main experiment (i.e., $4 \mathrm{~min}$ ). We collected and analyzed movement durations similar to the main experiment.

In all control experiments, the motor tasks, the experimental device, and the recording and analysis of kinematics and EMG signals were identical with the main experiment. The protocols of the control experiments are described in Results.

\section{Results}

\section{Main experiment}

\section{Right arm movements}

The mean duration of the fatiguing task was $4.05 \pm 1.21 \mathrm{~min}$ for session Ia (mental movements) and $3.95 \pm 1.14$ min for session IIa (actual movements). There was no significant difference in the duration of the fatiguing task between the sessions Ia and IIa $(t=1.28, \mathrm{df}=7, p=0.24)$. To quantify muscle fatigue, we compared RMS and MF values between the first $10 \mathrm{~s}$ and the last $10 \mathrm{~s}$ of the fatiguing task. For both sessions, RMS values significantly increased (for all muscle comparisons: $t>-2.7, \mathrm{df}=7$, $p<0.05$ ) and MF values significantly decreased (for all muscle comparisons: $t>3.3$, df $=7, p<0.01$ ), indicating the development of muscle fatigue (see Fig. 2 for a typical illustration). At the end of the fatiguing exercises, all the participants reported maximal perception of muscle fatigue ( score $=20$, on the Borg scale).

Figure 3 shows average durations for mental $(A)$ and actual $(B)$ right arm trials during prefatigue and postfatigue tests. ANOVA revealed an interaction effect between movement type and trials $\left(F_{(31,217)}=2.79, p<0.0001\right)$. Mental and actual durations were similar and stable from trial to trial during prefatigue (for all post hoc comparisons: $p>0.1$ ). Muscle fatigue significantly influenced the durations of mental trials (Fig. $3 A$ ). The first 


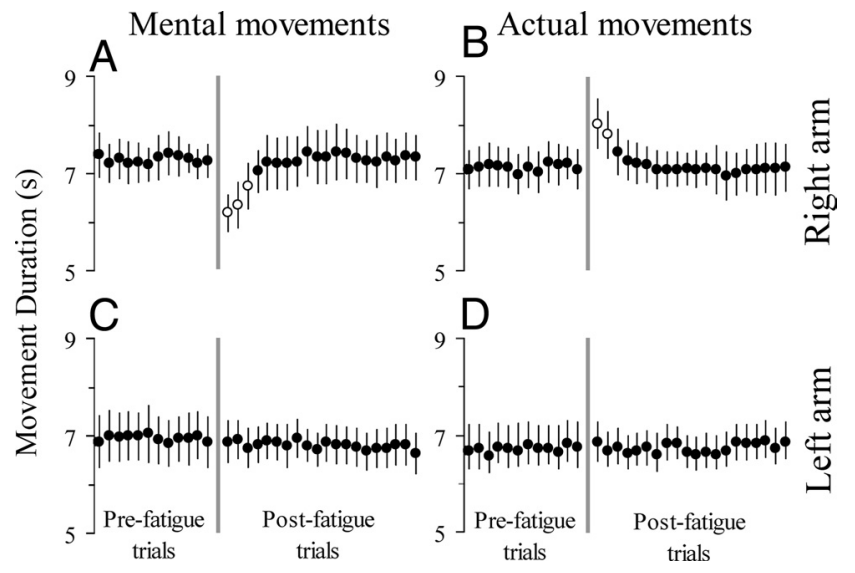

Figure 3. Main experiment. Average durations ( \pm SE) of mental $(\boldsymbol{A}, \boldsymbol{C})$ and actual $(\boldsymbol{B}, \boldsymbol{D})$ arm movements are shown for the prefatigue and the postfatigue tests. Trials depicted by white dots in postfatigue test indicate that these trials were significantly different from trials in the prefatigue test. In addition, the first three postfatigue actual trials were significantly longer than the first three postfatigue mental trials.

three postfatigue mental trials were significantly shorter than the prefatigue mental trials (for all comparisons: $p<0.05$ ). With respect to the average prefatigue mental duration $(7.28 \mathrm{~s})$, the decrease in duration for the first three postfatigue mental trials was $-15 \%,-12.7 \%$, and $-7.5 \%$, respectively. From the fourth postfatigue trial, mental durations returned to prefatigue values (for all comparisons: $p>0.1$ ). Muscle fatigue affected actual durations as well (Fig. $3 B$ ). Contrary to mental trials, the first two postfatigue actual trials were significantly longer than the prefatigue actual trials (for all comparisons: $p<0.05$ ). With respect to the average prefatigue actual duration (7.13 s), the decrease in duration for the first two postfatigue actual trials was $12.6 \%$ and $9.4 \%$, respectively. From the third postfatigue trial, actual durations returned to the prefatigue values (for all comparisons: $p>$ 0.1 ). Last, the first three postfatigue actual trials were significantly longer than the first three postfatigue mental trials (for all comparisons: $p<0.02$ ). From the fourth postfatigue trial, mental and actual durations were not significantly different (for all trial comparisons: $p>0.1$.

\section{Left arm movements}

The mean duration of the fatiguing task was $3.84 \pm 1.49 \mathrm{~min}$ for session Ib (mental movements) and $3.92 \pm 1.23$ min for session IIb (actual movements). There was no significant differences in the duration of the fatiguing task between the two sessions ( $t=$ -0.30 , df $=7, p=0.76$ ). To quantify muscle fatigue, we compared RMS and MF values between the first $10 \mathrm{~s}$ and the last $10 \mathrm{~s}$ of the fatiguing task. For both sessions, RMS values significantly increased (for all muscle comparisons: $t>-2.7, \mathrm{df}=7, p<0.05$ ) and MF values significantly decreased (for all muscle comparisons: $t>3.3 \mathrm{df}=7, p<0.01$ ), indicating the development of muscle fatigue. At the end of the fatiguing exercises, all the participants reported maximal perception of muscle fatigue (score $=$ 20 , on the Borg scale).

Figure 3 illustrates average durations of mental $(C)$ and actual (D) left arm trials during prefatigue and postfatigue tests. It is evident that muscle fatigue of the right arm did not influence mental and actual durations of the left arm. ANOVA showed that neither movement type $(p=0.2)$, nor trials $(p=0.98)$, nor their interaction $(p=0.57)$ reached significance.
EMG analysis of mental arm movements

We confirmed with surface EMG recording that there was no arm muscle activation; i.e., that participants performed purely mental movements. The statistical analysis revealed that right and left arm muscles remained silent during all mental trials (for all comparisons: $t<1.3$, df $=7, p>0.05$ ).

Kinematic and EMG features of the first postfatigue trial Figure 4 illustrates typical fingertip tangential velocity profiles for the six arm movements of the first prefatigue (gray traces) and the first postfatigue (black traces) trial. It clearly appears that the first postfatigue arm movement was faster (larger peak velocity and shorter movement duration) than the first prefatigue arm movement (see M1 in Fig. 4). For the other five arm movements (see M2-M6 in Fig. 4), an opposite effect was observed. Figure $5 \mathrm{~A}$ shows the average values of peak velocities for the six arm movements of the first postfatigue trial. These values were normalized with respect to the average value of the prefatigue trials. $t$ test comparisons between the first postfatigue trial and the average value of the prefatigue trials showed significant differences for the six movements that composed the trial (for all comparisons: $t>$ 3.5, $\mathrm{df}=7, p<0.01)$.

To quantify muscle activation patterns immediately after fatigue, we analyzed the EMG signals of agonist and antagonist muscles during the six arm movements of the first postfatigue trial. Figure $5 B$ shows the average duration of agonist and antagonist bursts, while Figure $5 C$ shows the average RMS of agonist bursts. The values reported in the Figure 5, $B$ and $C$, were normalized with respect to the average values of the prefatigue trials. It is noticeable that the duration of agonist and antagonist bursts and the RMS of agonist bursts during the first postfatigue arm movement were, respectively, shorter and greater compared to prefatigue (M1). The opposite result was obtained for the following 5 arm movements (M2-M6). $t$ tests, comparing agonist and antagonist durations, as well as RMS of agonist bursts, between the first postfatigue trial and the average values of prefatigue trials, showed significant differences for the six movements of the trial (for all comparisons: $t>2.9, \mathrm{df}=7, p<0.05$ ).

\section{Postfatigue adaptation}

The duration needed for returning to the prefatigue values after the end of the fatiguing exercise was on average $27.7 \pm 3.3 \mathrm{~s}$ for the mental trials and $21.8 \pm 2.8 \mathrm{~s}$ for the actual trials $(t=4.54, \mathrm{df}=7$, $p<0.001)$. Table 1 shows the temporal delays for each participant. It is noticeable that the return to the prefatigue values was longer for mental than actual trials for all the participants.

\section{Control experiments}

In the first control experiment, we examined whether postfatigue adaptation of mental arm movements (i.e., the time needed to return to prefatigue values after the fatiguing exercise) evolved in parallel with a natural recovering from fatigue. A progressive recovering from fatigue was observed for all arm muscles. RMS and MF values in prefatigue test were significantly different from the postfatigue short time interval and the postfatigue medium time interval (for all muscle comparisons: $t>3.1$, df $=7, p<$ 0.05 ), but not from the postfatigue long time interval (for all muscle comparisons: $t<1.5 \mathrm{df}=7, p>0.05)$. Figure 6 shows, as an example, the average RMS and MF values for the deltoid anterior (DA) muscle (RMS and MF of the other muscles qualitatively showed similar patterns of recovery).

In the second control experiment, we verified that postfatigue adaptation was qualitatively similar between actual and mental trials. Participants showed performances qualitatively similar to 

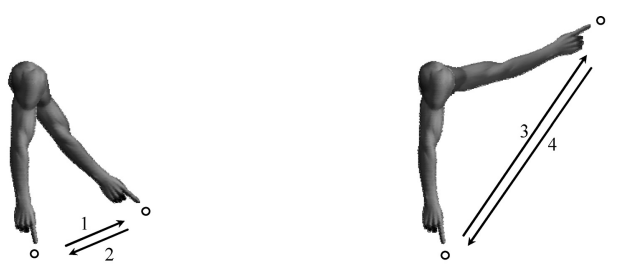

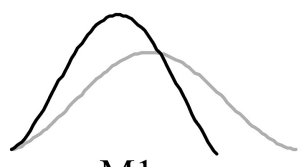

M1

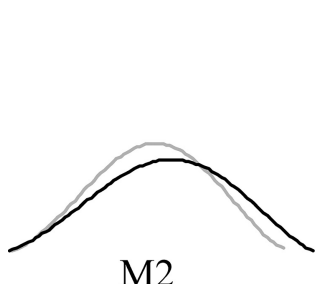

M2

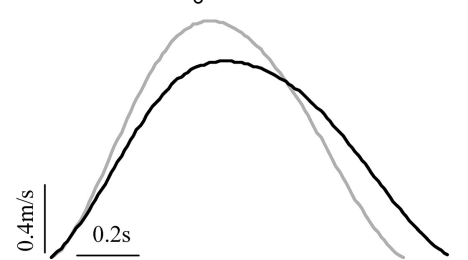

M3

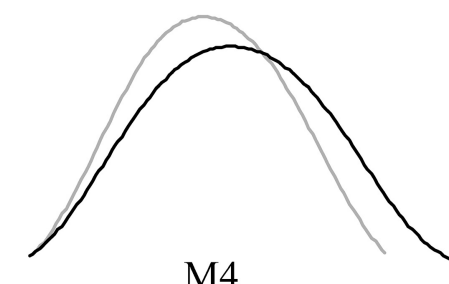

M4

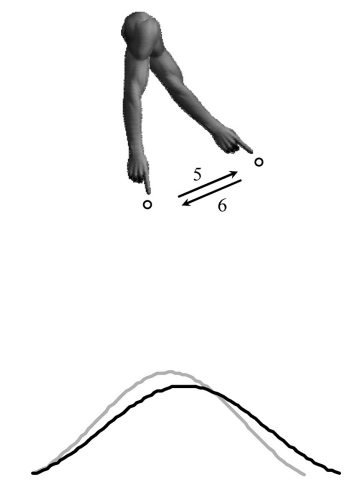

M5

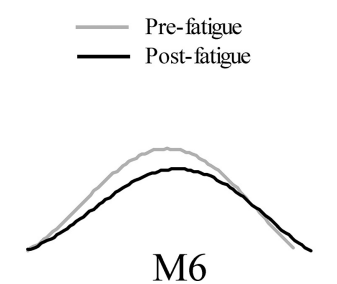

M6

Figure 4. Main experiment. Participants' initial and final positions during right arm movements (right-side view). Typical fingertip tangential velocity profiles are depicted for the six arm movements (M1-M6) of the first prefatigue (gray traces) and the first postfatigue (black traces) trial.

those of the main experiment. Notably, there was an increase in actual duration (black bar) and a decrease in mental duration (white bar) immediately after fatigue (Fig. 7A). The duration of the fifth postfatigue actual trial, either after four mental trials (session I) or four actual trials (session II), was similar with that of the fist prefatigue trial (see gray bars). The statistical analysis (Wilcoxon tests) showed that the first postfatigue trial was significantly different from the first prefatigue trial $(Z>2, \mathrm{df}=4, p<$ $0.05)$ and from the fifth postfatigue trial $(Z>2, \mathrm{df}=4, p<0.05)$. Figure $7 B$ depicts the duration of each movement (M1-M6) of the first prefatigue and the fifth postfatigue trial as a percentage of their total movement duration. This normalization permits to compare the internal structure of the trial independently of movement duration. It appears that the temporal structure of these trials is approximately similar before and after fatigue. This suggests a complete recovery at the kinematic level after four mental (session I) or four actual (session II) postfatigue trials. The analysis of the timing of agonist and antagonist muscle activation patterns further supports this observation. For simplicity reasons, we illustrate the EMG patterns for the first movement of the first prefatigue trial and the first movement of the fifth postfatigue trial (Fig. $7 C, D$ ). It appears that the timing of muscle activation did not differ between actual and mental movements and between prefatigue and postfatigue trials.

In the third control experiment, we tested whether temporal differences between prefatigue and postfatigue actual or mental trials, observed in the main experiment, were due to variations in arm postures. Figure $8, A$ and $B$, illustrates trial-by-trial the average durations for mental and actual right arm movements during pretest and posttest. Passive position of the right arm did not influence mental and actual movement durations. ANOVA showed that movement type $(p=0.2)$, trials $(p=0.99)$, and their interaction $(p=0.59)$ did not reach significance.

\section{Discussion}

We investigated to what extent muscle fatigue could influence the content of actual and mental actions. Our findings revealed that muscle fatigue significantly altered the neural drives sent to the fatigued arm muscles. This significantly biased motor prediction and, therefore, drastically modified the temporal features of both mental and actual movements. This effect was specific to the fatigued arm, since actual and mental movements of the contralateral nonfatigued arm were not affected. Last, recovering from muscle fatigue was faster for actual than mental arm movements.

\section{Temporal similarities between actual and mental arm movements before muscle fatigue}

We found robust temporal correspondences between actual and mental movements, accomplished either with the right or the left arm, before muscle fatigue. These findings corroborate the premise that actual and mental actions share common neural and cognitive processes (Sirigu et al., 1996; Jeannerod, 2001; Gueugneau and Papaxanthis, 2010). In our study, the temporal matching between actual and mental movements and their consistency from trial to trial before fatigue guarantee that participants mentally simulated arm movements with high accuracy and precision. The involvement of internal predictive models in both actual and mental movements explains their robust isochrony and reliability from trial to trial (Gentili et al., 2010). During an actual movement, the internal forward model relates the sensory signals of the actual state of the arm (e.g., position, time, and velocity) to the prepared motor commands and predicts the future states of the arm. During motor imagery, neural commands are prepared, but are blocked before reaching muscle level; i.e., no movement occurs. However, a copy of these motor commands is available to the forward model, which predicts the future states of the arm and provides temporal information very similar to that of actual movements.

\section{Muscle fatigue alters the temporal features of actual and mental arm movements}

We found that muscle fatigue transiently influenced the control of vertical arm movements. Actual movements were in general slower immediately after fatigue, but progressively returned to prefatigue values. These findings broaden those of previous studies, which have shown that fatigue modifies the timing of muscle activation (Corcos et al., 2002), changes the intersegmental coordination of the arm (Forestier and Nougier, 1998; Huffenus et al., 2006), alters the equilibrium and the joint coordination strategies during a whole-body reaching task (Schmid et al., 2006), and affects position, movement, and effort sense (Gandevia, 2001; Walsh et al., 2010).

The most salient result in the current study was the effects of muscle fatigue on mental actions. Mental movements were faster immediately after fatigue and gradually returned to prefatigue 


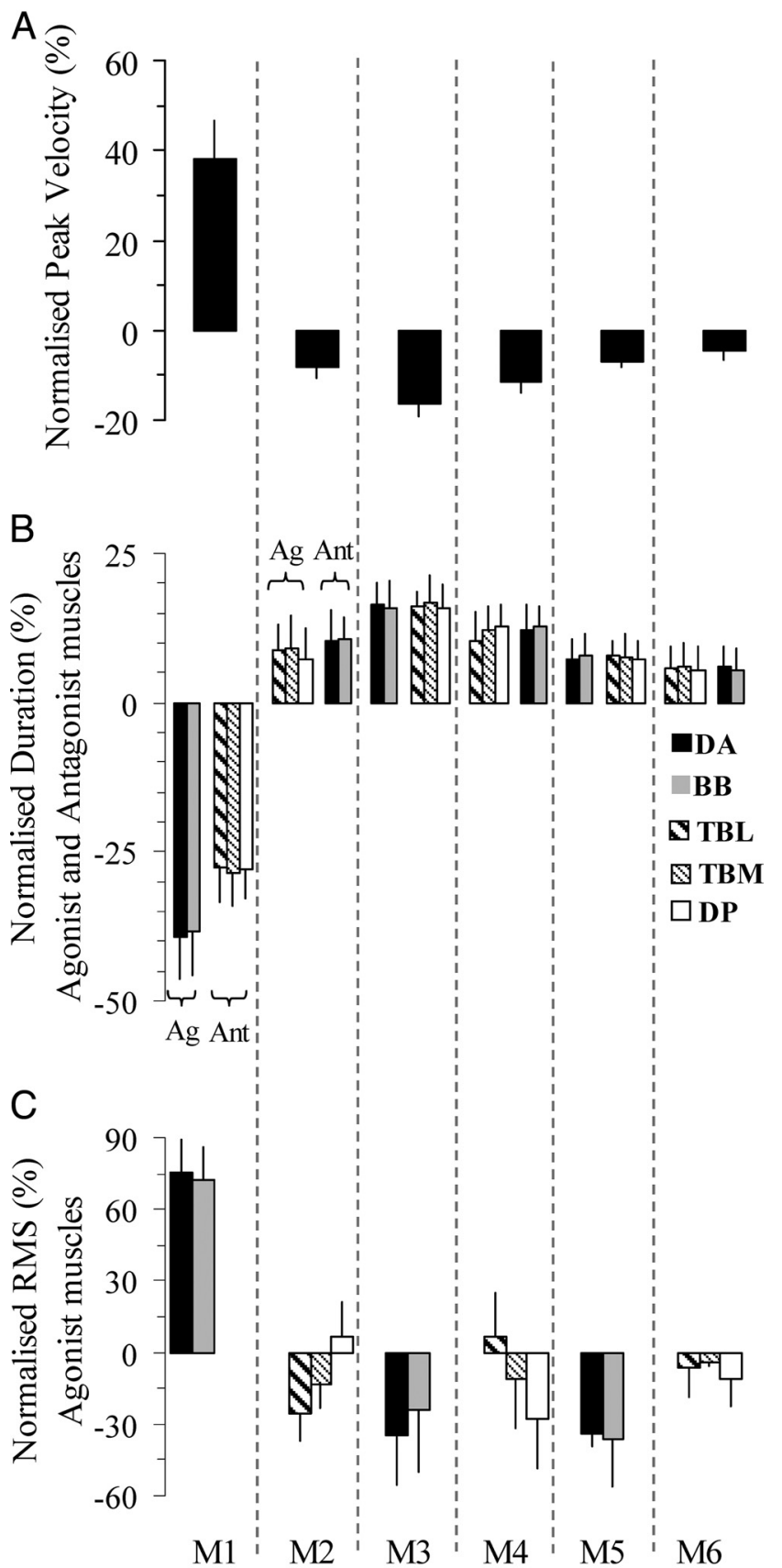

\section{First post-fatigue trial}

Figure 5. Main experiment. $A$, Average-normalized (with respect to the average prefatigue trials) peak velocities ( $\pm S D$ ) of the six arm movements of the first postfatigue trial. $B$, Averagenormalized (with respect to the average prefatigue trials) durations ( \pm SD) of agonist and antagonist muscle activation for the six arm movements of the first postfatigue trial. $\mathbf{C}$, Average-normalized (with respect to the average prefatigue trials) RMS values $( \pm S D$ ) of each agonist muscle for the six arm movements of the first postfatigue trial. DA and BB are agonist muscles during upward movements (M1, M3, and M5), while DP, TBL, and TBM are agonist muscles during downward movements (M2, M4, and M6). M1-M6 indicate the six movements that composed the trials.

values. This is an original finding and puts forward the idea that action representations are embodied and that they integrate the current state of the motor system. Previous and current findings seem to indicate that action context is integrated into
Table 1. Duration of postfatigue adaptation

\begin{tabular}{lll}
\hline & \multicolumn{2}{l}{ Time of postfatigue adaptation $(\mathrm{s})$} \\
\cline { 2 - 3 } Participants & Mental & Actual \\
\hline P1 & 33.62 & 24.85 \\
P2 & 28.65 & 26.89 \\
P3 & 27.94 & 18.34 \\
P4 & 29.67 & 17.89 \\
P5 & 21.12 & 17.53 \\
P6 & 25.67 & 21.77 \\
P7 & 27.11 & 22.89 \\
P8 & 27.59 & 24.39 \\
\hline
\end{tabular}

Individual durations for returning to the prefatigue values after the end of the fatiguing exercise are reported for actual and mental trials.
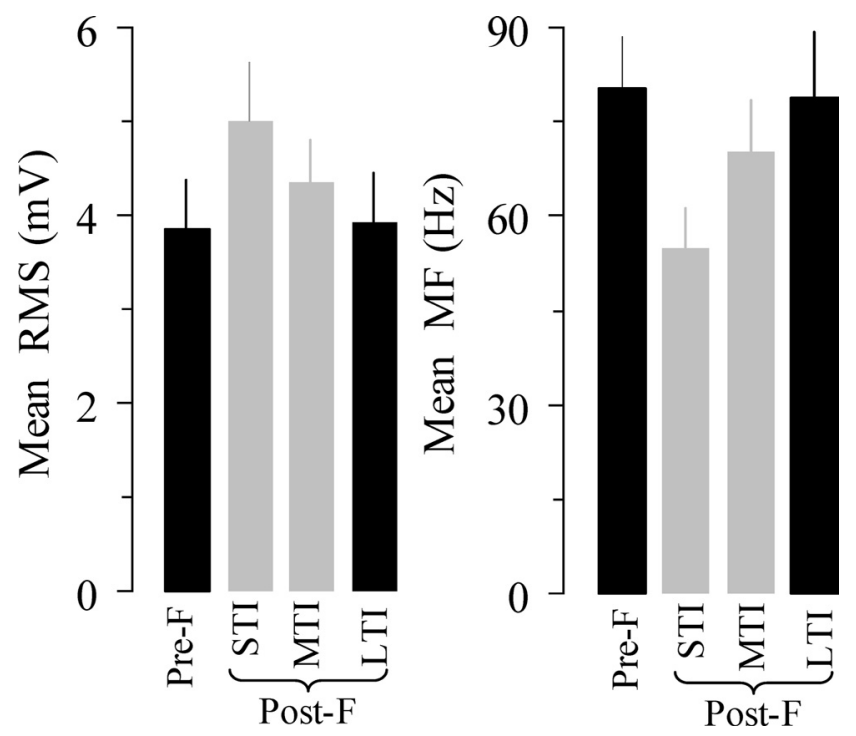

Figure 6. Control experiment. Average RMS and MF values from the muscle DA are depicted for the prefatigue (Pre-F) and the three postfatigue tests (Post-F). RMS and MF values depicted by gray bars are significantly different from those depicted by black bars. STI, Short time interval; MTI, middle time interval; LTI, long time interval.

mental states. Vargas et al. (2004) found that corticospinal excitability is enhanced when the compatibility between the actual hand posture and the imagined movement is maximized. In addition, Sirigu and Duhamel (2001) showed that changing the actual posture of the hand affects the ease with which mental representations are generated. We also observed that muscle fatigue specifically-and not generally-affects mental actions. Only mental durations of the fatigued arm were modified; those of the nonfatigued arm were not. This result suggests that the bias of muscle fatigue on mental durations cannot be explained in terms of nonmotor phenomena (namely cognitive effort, or general perception of fatigue), but likely in terms of sensorimotor state estimation and prediction. Previous studies have argued in favor of effector specificity on mental actions. Fadiga et al. (1999) showed that the modulation of corticospinal excitability during motor imagery reflects precisely the motor action being imagined. In their study, motor imagery of forearm flexion, but not motor imagery of forearm extension, enhances biceps brachial MEPs. Ehrsson et al. (2003) have demonstrated that mental actions of several body parts engage the somatotopically organized sections of the primary motor cortex in a systematic manner. This suggests that content of the mental motor image (i.e., the body part) is reflected in the pattern of motor cortical activation. 


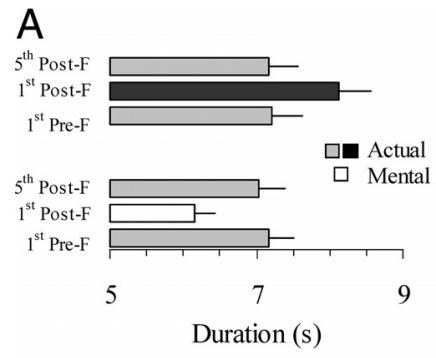

B

C

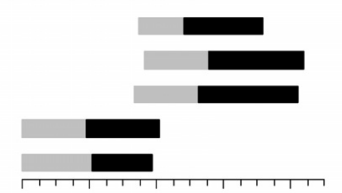

$\begin{array}{lllll}0 & 0.2 & 0.4 & 0.6 & 0.8\end{array}$

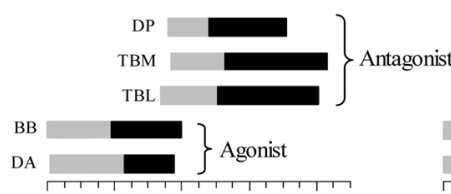

\begin{tabular}{lllll}
0 & 0.2 & 0.4 & 0.6 & 0.8 \\
& & \\
& \multicolumn{4}{c}{ Duration (s) }
\end{tabular}

\begin{tabular}{ccccc}
0.0 & 0.2 & 0.4 & 0.6 & 0.8 \\
\multicolumn{4}{c}{ Duration (s) }
\end{tabular}

Figure 7. Control experiment. $\boldsymbol{A}$, Average durations ( $\pm S D$ ) of the first prefatigue, the first postfatigue, and the fifth postfatigue trial for the sessions I and II. $\boldsymbol{B}$, Average duration of each movement (M1-M6) of the first prefatigue and the fifth postfatigue trial as a percentage of their total movement duration. $C, D$, The timing of agonist (DA and $B B$ ) and antagonist (DP, TBL, and TBM) muscle activation patterns for session I ( $\boldsymbol{C}$ and session II (D). For each muscle, gray bars show the duration from EMG initiation to peak EMG, while black bars show the duration from peak EMG to the end of the EMG pattern.

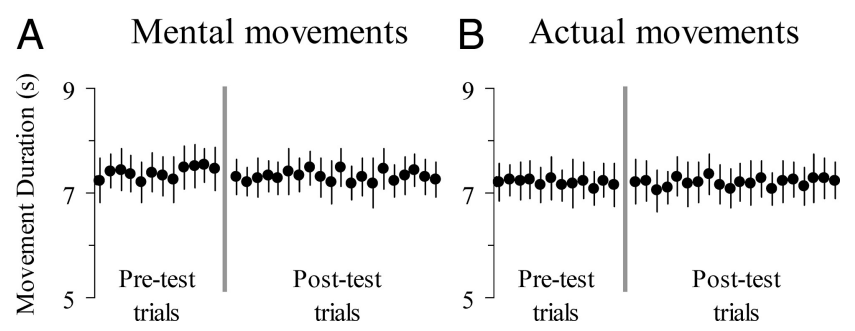

Figure 8. Control experiment. Average durations ( \pm SE) of mental $(\boldsymbol{A})$ and actual $(\boldsymbol{B})$ right arm movements are depicted before (pretest) and after (posttest) a passive task, during which the right arm was passively maintained at the horizontal level. This task replaced the fatiguing task of the main experiment.

\section{Adaptation of actual and mental arm movements after muscle fatigue}

A main question in our study concerns the underling mechanisms by which muscle fatigue influences mental and actual arm movements. Motor efferent outflow signals, sensorimotor prediction, and the theory of internal models (Wolpert and Miall, 1996; Wolpert and Ghahramani, 2000) are the key notions for interpreting our results.

We observed that the first actual movement in the postfatigue trial was dramatically faster than the first actual movement in the prefatigue trial due to larger motor commands sent to arm muscles. This finding suggests that the CNS overestimated the motor drive necessary to displace the fatigued arm, and that the motor planning was inappropriate. Immediately after this erroneous intention, the CNS progressively adapted the speed of the move- ment by modifying the neural drives sent to the muscles until complete adaptation of movement duration (i.e., return to prefatigue values). Internal model theory postulates that forward internal models mimic the causal flow of the physical process by predicting the future sensorimotor state (e.g., position and velocity) given the efferent copy of the motor command and the current state (Wolpert and Flanagan, 2001). During the first actual movement after fatigue, state estimation provided by the forward model (based on the variable current state of the fatigued arm and the inappropriate large efferent copy) did not match the actual state of the arm provided by the sensory feedback. Forward model predictions about the consequence of motor commands were then combined with sensory feedback to provide accurate estimations of the state of the motor system (Wolpert et al., 1995; Kawato, 1999). Therefore, the discrepancy between the estimated and the actual state of the arm was used as an error signal to change the plan of the movement and to drive the adaptation of the future movements.

Mental and actual movements share similar motor representations and intentions (Jeannerod, 2001). Therefore, state estimation, based on forward internal model output, is a common process during actual and mental actions (Wolpert and Flanagan, 2001; Gentili et al., 2010). We assume that during mental arm movements after fatigue, the brain prepares a large motor command, as it does for actual movements, but this motor command is blocked before reaching muscle level (we did not observe any EMG activity during mental movements). However, its efferent copy is available to the forward model, which naturally predicts very fast movements. Since during mental movements there is not sensory information, and state estimation derives from forward model alone, the brain continues to simulate faster mental movements after fatigue based on inappropriate (large) neural drives. This explains why mental movements were consistently faster than actual movements after fatigue, at least until their complete adaptation.

This process, i.e., state estimation without sensory feedback, also explains the slower update of motor controller (i.e., motor commands) during mental compared to actual movements after fatigue. Slower adaptation is a general trend of mental state because it has also been observed during mental training. Due to trial-by-trial variability in the state estimation during mental practice, the internal training signal used for learning is itself variable and leads to a slower update of the motor controller during mental training than during physical training (Gentili et al., 2010). In our study, mental arm movements after fatigue gradually returned to the prefatigue values. Adaptation of mental movements, although longer than with actual movements, was complete (Fig. 7) and probably due to a natural recovery from fatigue (Fig. 6).

Our result of a larger efferent motor outflow after fatigue is consistent with previous findings, which showed that alterations in force estimation during fatigue reflect changes in the descending voluntary command (Gandevia, 2001; Takahashi et al., 2006). Neuroimaging studies have also shown increased cortical activation of the contralateral primary motor (MI) and the primary and secondary somatosensory (SI and SII) areas with the duration of muscle contraction (Liu et al., 2003; Korotkov et al., 2005; Post et al., 2009). In addition, previous studies showed that outflow signals play an important role on position and movement sense (Gandevia et al., 1996). Walsh et al. (2010) found that motor command signals can generate graded sensations of continuous movement in the absence of sensory input. Subjects perceived 
their phantom wrist to move faster if they made bigger efforts and further if they made longer efforts.

In synopsis, we found that mental representations of actions are altered by muscle fatigue. Our findings and complementary data from several studies, which showed that mental fatigue influences motor performance and action planning (Lorist et al., 2000, 2002; Marcora et al., 2009), underline the interdependence of motor and cognitive states. Because mental training is consistently used, alone or in combination with physical training, in sports and motor rehabilitation, we consider that sport psychologists and therapists should take into consideration physical and/or mental fatigue in the elaboration of their training programs.

\section{References}

Bakker M, de Lange FP, Stevens JA, Toni I, Bloem BR (2007) Motor imagery of gait: a quantitative approach. Exp Brain Res 179:497-504.

Corcos DM, Jiang HY, Wilding J, Gottlieb GL (2002) Fatigue induced changes in phasic muscle activation patterns for fast elbow flexion movements. Exp Brain Res 142:1-12.

Courtine G, Papaxanthis C, Gentili R, Pozzo T (2004) Gait-dependent motor memory facilitation in covert movement execution. Brain Res Cogn Brain Res 22:67-75.

Demougeot L, Normand H, Denise P, Papaxanthis C (2009) Discrete and effortful imagined movements do not specifically activate the autonomic nervous system. PLoS One 4:e6769.

Ehrsson HH, Geyer S, Naito E (2003) Imagery of voluntary movement of fingers, toes, and tongue activates corresponding body-part-specific motor representations. J Neurophysiol 90:3304-3316.

Fadiga L, Craighero L (2004) Electrophysiology of action representation. J Clin Neurophysiol 21:157-169.

Fadiga L, Buccino G, Craighero L, Fogassi L, Gallese V, Pavesi G (1999) Corticospinal excitability is specifically modulated by motor imagery: a magnetic stimulation study. Neuropsychologia 37:147-158.

Forestier N, Nougier V (1998) The effects of muscular fatigue on the coordination of a multijoint movement in human. Neurosci Lett 252:187-190.

Gandevia SC (2001) Spinal and supraspinal factors in human muscle fatigue. Physiol Rev 81:1725-1789.

Gandevia SC, Allen GM, Butler JE, Taylor JL (1996) Supraspinal factors in human muscle fatigue: evidence for suboptimal output from the motor cortex. J Physiol 490:529-536.

Gentili R, Papaxanthis C, Pozzo T (2006) Improvement and generalization of arm motor performance through motor imagery practice. Neuroscience 137:761-772.

Gentili R, Han CE, Schweighofer N, Papaxanthis C (2010) Motor learning without doing: trial-by-trial improvement in motor performance during mental training. J Neurophysiol 104:774-783.

Gueugneau N, Papaxanthis C (2010) Time-of-day effects on the internal simulation of motor actions: psychophysical evidence from pointing movements with the dominant and non-dominant arm. Chronobiol Int 27:620-639.

Gueugneau N, Crognier L, Papaxanthis C (2008) The influence of eye movements on the temporal features of executed and imagined arm movements. Brain Res 1187:95-102.

Gueugneau N, Mauvieux B, Papaxanthis C (2009) Circadian modulation of mentally simulated motor actions: implications for the potential use of motor imagery in rehabilitation. Neurorehabil Neural Repair 23:237-245.

Hall CR, Martin KA (1997) Measuring movement imagery abilities: a revision of the movement imagery questionnaire. J Ment Imag 21:143-154.

Huffenus AF, Amarantini D, Forestier N (2006) Effects of distal and proximal arm muscles fatigue on multi-joint movement organization. Exp Brain Res 170:438-447.
Jeannerod M (2001) Neural simulation of action: a unifying mechanism for motor cognition. Neuroimage 14:S103-S109.

Jurell KC (1998) Surface EMG and fatigue. Phys Med Rehabil Clin N Am 9:933-947, viii-ix.

Kawato M (1999) Internal models for motor control and trajectory planning. Curr Opin Neurobiol 9:718-727.

Komi PV, Tesch P (1979) EMG frequency spectrum, muscle structure, and fatigue during dynamic contractions in man. Eur J Appl Physiol Occup Physiol 42:41-50.

Korotkov A, Radovanovic S, Ljubisavljevic M, Lyskov E, Kataeva G, Roudas M, Pakhomov S, Thunberg J, Medvedev S, Johansson H (2005) Comparison of brain activation after sustained non-fatiguing and fatiguing muscle contraction: a positron emission tomography study. Exp Brain Res 163:65-74.

Liu JZ, Shan ZY, Zhang LD, Sahgal V, Brown RW, Yue GH (2003) Human brain activation during sustained and intermittent submaximal fatigue muscle contractions: an FMRI study. J Neurophysiol 90:300-312.

Lorist MM, Klein M, Nieuwenhuis S, De Jong R, Mulder G, Meijman TF (2000) Mental fatigue and task control: planning and preparation. Psychophysiology 37:614-625.

Lorist MM, Kernell D, Meijman TF, Zijdewind I (2002) Motor fatigue and cognitive task performance in humans. J Physiol 545:313-319.

Marcora SM, Staiano W, Manning V (2009) Mental fatigue impairs physical performance in humans. J Appl Physiol 106:857-864.

Miall RC, Weir DJ, Wolpert DM, Stein JF (1993) Is the cerebellum a Smith predictor? J Mot Behav 25:203-216.

Munzert J, Lorey B, Zentgraf K (2009) Cognitive motor processes: the role of motor imagery in the study of motor representations. Brain Res Rev 60:306-326.

Oldfield RC (1971) The assessment and analysis of handedness: the Edinburgh inventory. Neuropsychologia 9:97-113.

Post M, Steens A, Renken R, Maurits NM, Zijdewind I (2009) Voluntary activation and cortical activity during a sustained maximal contraction: an fMRI study. Hum Brain Mapp 30:1014-1027.

Ranganathan VK, Siemionow V, Liu JZ, Sahgal V, Yue GH (2004) From mental power to muscle power-gaining strength by using the mind. Neuropsychologia 42:944-956.

Schmid M, Schieppati M, Pozzo T (2006) Effect of fatigue on the precision of a whole-body pointing task. Neuroscience 139:909-920.

Sirigu A, Duhamel JR (2001) Motor and visual imagery as two complementary but neurally dissociable mental processes. J Cogn Neurosci 13:910-919.

Sirigu A, Duhamel JR, Cohen L, Pillon B, Dubois B, Agid Y (1996) The mental representation of hand movements after parietal cortex damage. Science 273:1564-1568.

Skoura X, Personnier P, Vinter A, Pozzo T, Papaxanthis C (2008) Decline in motor prediction in elderly subjects: right versus left arm differences in mentally simulated motor actions. Cortex 44:1271-1278.

Takahashi CD, Nemet D, Rose-Gottron CM, Larson JK, Cooper DM, Reinkensmeyer DJ (2006) Effect of muscle fatigue on internal model formation and retention during reaching with the arm. J Appl Physiol 100:695-706.

Vargas CD, Olivier E, Craighero L, Fadiga L, Duhamel JR, Sirigu A (2004) The influence of hand posture on corticospinal excitability during motor imagery: a transcranial magnetic stimulation study. Cereb Cortex 14:1200-1206.

Walsh LD, Gandevia SC, Taylor JL (2010) Illusory movements of a phantom hand grade with the duration and magnitude of motor commands. J Physiol 588:1269-1280.

Wolpert DM, Flanagan JR (2001) Motor prediction. Curr Biol 11:R729-R732.

Wolpert DM, Ghahramani Z (2000) Computational principles of movement neuroscience. Nat Neurosci 3 [Suppl]:1212-1217.

Wolpert DM, Miall RC (1996) Forward models for physiological motor control. Neural Netw 9:1265-1279.

Wolpert DM, Ghahramani Z, Jordan MI (1995) An internal model for sensorimotor integration. Science 269:1880-1882. 